\title{
Organização da atenção à saúde bucal prestada ao idoso nas equipes de saúde bucal da Estratégia Saúde da Família
}

\author{
Organization of oral health care to the elderly in the Family Health Strategy
}

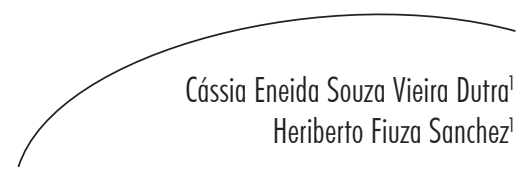

\section{Resumo}

Este trabalho teve por objetivo realizar uma revisão de literatura sobre a atenção à saúde bucal do idoso, considerando a necessidade de organizar a atenção à saúde bucal para essa faixa etária no cotidiano da Estratégia Saúde da Família. Os dados foram buscados, através da BIREME (Biblioteca Virtual em Saúde-BVS), nas bases de dados BBO, MEDLINE e LILACS, em publicações datadas entre 2000 e 2010, na língua portuguesa. Foram encontradas 46 publicações. Também foram pesquisados documentos oficiais, envolvendo políticas de saúde do idoso. Aplicados os critérios de inclusão e exclusão, restaram 23 publicações científicas para análise. Os artigos selecionados confirmam a necessidade de organizar a atenção à saúde bucal do idoso, pois estudos epidemiológicos apontam para a precária condição de saúde bucal desse grupo populacional. A organização da atenção à saúde bucal passa pela necessidade de um planejamento inter e multidisciplinar. A intersetorialidade pode contribuir sobremaneira para a promoção da saúde.

\section{Abstract}

This study aimed to conduct a literature review on the oral health of the elderly, considering the need to organize oral health care for this age group in the routine of the Family Health Strategy. Data were collected, through BIREME (Biblioteca Virtual na Saúde-BVS), in databases BBO, LILACS and MEDLINE, in publications dated between 2000 and 2010, in Portuguese. We selected 46 publications. Official documents, policies involving health of the elderly were also surveyed. Inclusion and exclusion criteria were applied and 23 scientific publications were selected for analysis. The articles confirm the need to organize oral health care of the elderly. Such organization requires the involvement of several specialties, not only due to the physiological process of aging, but also because in most cases this public displays multiple systemic changes. The elderly care needs the involvement of a multidisciplinary and interdisciplinary team,

Palavras-chave: Idoso. Saúde Bucal do Idoso. Saúde Bucal. Atenção à Saúde. Atenção Primária à Saúde.
Key words: Elderly. Elderly's Oral Health. Oral Health. Health Care (Public Health). Primary Health Care. 
seeking health promotion and intersectoral support. Epidemiological studies indicate the poor oral health status of this population group. The organization of oral health care requires inter- and multi-disciplinary planning. Intersectoriality may contribute significantly to health promotion.

\section{INTRODUÇÃO}

Discutir a nova realidade demográfica brasileira se torna cada vez mais urgente, pois o grande crescimento da população idosa observado nos últimos anos deve ser levado em consideração no planejamento e reformulação das políticas social, econômica e de saúde. ${ }^{1}$

Além da transição demográfica, o Brasil tem experimentado uma transição epidemiológica, caracterizada pelo aumento de doenças crônicodegenerativas e redução das infecto-contagiosas. Como consequência, a demanda e custos dos serviços de saúde serão maiores. ${ }^{2,3}$

A saúde bucal do idoso foi submetida a um modelo assistencial curativo mutilador com características excludentes, muitas vezes restritos aos serviços de urgência odontológica. Esse modelo assistencial gerou um quadro de saúde bucal precária nos idosos, que apresentam alta prevalência de doenças bucais, como cáries, doenças periodontais, edentulismo e necessidade de uso de próteses. Assim, a saúde bucal do idoso implica organizar e ampliar a oferta tanto de atenção preventiva e de promoção de saúde, como de atenção curativa e reabilitadora. ${ }^{3,4}$

Em 1994, o Ministério da Saúde adotou a Estratégia Saúde da Família (ESF), na época chamado Programa Saúde da Família, como uma estratégia prioritária para a organização da atenção primária e estruturação do sistema de saúde. A ESF trabalha com práticas interdisciplinares desenvolvidas por equipes que se responsabilizam pela saúde da população a ela adscrita e na perspectiva de uma atenção integral humanizada, considerando a realidade local e valorizando as diferentes necessidades dos grupos populacionais. ${ }^{5}$

Decorridos seis anos da criação da ESF, o Ministério da Saúde (MS), com o objetivo de melhorar os índices epidemiológicos de saúde bucal e ampliar o acesso da população brasileira às ações de promoção, prevenção e recuperação da saúde em todos os níveis de atenção, regulamentou a Portaria no. 1.444/GM, de 28 de dezembro de 2000, que aprovou as normas e diretrizes de inclusão da saúde bucal na ESF. Criou-se, assim, o incentivo destinado ao financiamento de ações e à inserção de profissionais da área odontológica. ${ }^{6}$

Em 2004, o governo federal criou a Política Nacional de Saúde Bucal (PNSB), também chamada Programa Brasil Sorridente. Suas diretrizes têm o conceito do cuidado com eixo norteador de reorientação do modelo, deixando a concepção de saúde não centrada na doença, mas, sobretudo, na promoção de qualidade de vida e intervenção nos fatores de risco, com a incorporação de ações programáticas de forma mais abrangente e desenvolvimento de ações intersetoriais. No campo de ação da assistência, essas diretrizes apontam para a ampliação e a qualificação da atenção primária, possibilitando o acesso a todas as faixas etárias e à oferta de mais serviços. Buscando a integralidade da atenção, são assegurados atendimentos nos níveis secundário e terciário.?

O objetivo deste trabalho foi realizar uma revisão de literatura sobre a atenção à saúde bucal do idoso, possibilitando que sejam elaboradas 
propostas voltadas para o planejamento da atenção a saúde bucal desse grupo populacional no cotidiano das Equipes de Saúde da Família, contribuindo, assim, para a consolidação das ações do Sistema Único de Saúde (SUS).

\section{METODOLOGIA}

Trata-se de revisão narrativa, considerada apropriada para descrever e discutir o desenvolvimento ou "estado da arte" de determinado assunto, sob o ponto de vista teórico ou contextual. Esse tipo de revisão permite adquirir e atualizar o conhecimento sobre determinada temática específica, sendo ainda caracterizada pela análise da literatura publicada em livros, teses, dissertações, monografias e artigos em formato impresso ou eletrônico. ${ }^{8}$

Para obtenção dos dados, utilizou-se levantamento bibliográfico realizado na BIREME (Biblioteca Virtual em Saúde-BVS) nas bases de dados BBO, MEDLINE e LILACS. Foram incluídas publicações datadas entre 2000 e 2010, na língua portuguesa, na forma de artigo, tendo sido usados inicialmente os unitermos "saúde bucal idoso" e "atenção à saúde bucal".

Além da pesquisa na BIREME, foram pesquisados documentos oficiais do governo federal, envolvendo políticas e saúde do idoso e cadernos da atenção básica, saúde bucal e saúde do idoso. A busca das publicações no Ministério da Saúde (MS) foi feita por meio do site desse órgão.*

Considerando todas as bases, foram recuperadas 46 produções científicas, dentre artigos, monografias e teses. Para prosseguimento

\footnotetext{
*http://dtr2001.saude.gov.br/editora/produtos/livros/genero/ livros. As publicações pesquisadas foram: Caderno de Atenção Básica: Saúde Bucal, Cadernos de Atenção Básica: Envelhecimento da Pessoa Idosa, Linha-guia: Atenção em Saúde Bucal e Linha-guia: Atenção à Saúde do Idoso.
}

do estudo, o critério de seleção era que fossem artigos científicos, sendo selecionados 36 .

Procedeu-se inicialmente à leitura dos títulos e resumos desses artigos. Após esse segundo processo seletivo, restaram 23 artigos para leitura e análise. A leitura dos resumos levou em consideração a presença de conteúdo que abordasse a questão da atenção à saúde bucal para a população idosa e possibilidades de como organizar essa atenção à saúde no âmbito do SUS. O processo de exclusão levou em consideração a presença de conteúdo presente nos títulos e resumos, que, embora de interesse para a saúde bucal dos idosos (por exemplo, repercussões bucais de problemas sistêmicos), não fosse diretamente relacionada a propostas de organização da atenção à saúde bucal para essa faixa etária.

\section{RESULTADOS}

Observa-se que os artigos podem ser agregados em dois grupos principais, de acordo com a abordagem adotada nas pesquisas. Dessa maneira, 56,5\% $(n=13)$ dos artigos abordavam políticas voltadas para a saúde bucal de idosos, enquanto 43,5\% ( $\mathrm{n}=10)$ abordavam a mesma questão a partir de estudos epidemiológicos realizados em diferentes realidades do país. Quanto à metodologia empregada, observase predominância de estudos quantitativos em relação aos de abordagem qualitativa.

Como aspecto geral, é possível apontar que a saúde bucal do idoso brasileiro encontrase em situação precária, com elevados índices de edentulismo, refletindo a ineficácia historicamente presente nos serviços públicos de atenção odontológica, limitado a extrações em série e serviços de urgência, baseados no modelo curativista.

O quadro 1 traz uma exposição resumida do conteúdo presente nos artigos selecionados. 
Quadro 1. Autores, títulos, ano de publicação, objetivos e principais resultados dos trabalhos selecionados

\begin{tabular}{|c|c|}
\hline Autores, títulos e ano de publicação & Objetivos e principais resultados \\
\hline $\begin{array}{l}\text { Macedo et al. Proposta de um } \\
\text { protocolo para o atendimento } \\
\text { odontológico do paciente idoso na } \\
\text { atenção básica, } 2009 \text {. }\end{array}$ & $\begin{array}{l}\text { O objetivo deste estudo foi desenvolver um protocolo de atendimento } \\
\text { odontológico para o paciente idoso no programa de saúde da família, propondo } \\
\text { uma reorientação da prática dos procedimentos odontológicos, evidenciando } \\
\text { a importância do atendimento multidisciplinar, para prevenção, controle e } \\
\text { tratamento das suas afecções. }\end{array}$ \\
\hline $\begin{array}{l}\text { Martins et al. Autoavaliação de saúde } \\
\text { bucal em idosos: análise com base em } \\
\text { modelo multidimensional, } 2009 \text {. }\end{array}$ & $\begin{array}{l}\text { Propôs-se investigar fatores associados à autoavaliação negativa da saúde bucal. } \\
\text { Apesar das precárias condições bucais, a maioria autoavaliou positivamente sua } \\
\text { saúde bucal. Condições subjetivas estiveram mais fortemente associadas do que } \\
\text { as objetivas. Os resultados sugerem iniquidade e permitem orientar políticas } \\
\text { públicas que objetivem saúde bucal e qualidade de vida. }\end{array}$ \\
\hline $\begin{array}{l}\text { Costa, Maciel \& Cavalcanti. Acesso } \\
\text { aos serviços odontológicos e motivos } \\
\text { da procura por atendimento por } \\
\text { pacientes idosos em Campina } \\
\text { Grande-PB, 2008. }\end{array}$ & $\begin{array}{l}\text { Avaliar o acesso aos serviços odontológicos e os motivos da procura por } \\
\text { atendimento por idosos de Campina Grande-PB. Verifica-se necessidade de } \\
\text { adotar políticas de promoção de saúde direcionadas para a saúde bucal do idoso, } \\
\text { possibilitando uma reestruturação no processo de socialização desses indivíduos. }\end{array}$ \\
\hline $\begin{array}{l}\text { Martins et al. Uso de serviços } \\
\text { odontológicos por rotina entre idosos } \\
\text { brasileiros: Projeto SB Brasil, } 2008 .\end{array}$ & $\begin{array}{l}\text { Investigou-se o uso de serviços odontológicos por rotina entre idosos brasileiros } \\
\text { participantes do Projeto SB Brasil. Iniquidades, barreiras financeiras e falta } \\
\text { de informações parecem prejudicar o uso rotineiro, indicando necessidade de } \\
\text { políticas públicas. }\end{array}$ \\
\hline $\begin{array}{l}\text { Saintrain \& Vieira. Saúde bucal do } \\
\text { idoso: abordagem interdisciplinar, } \\
\text { 2008. }\end{array}$ & $\begin{array}{l}\text { Este texto objetiva ressaltar a importância da metodologia problematizadora no } \\
\text { contexto da interdisciplinaridade, em que as ações compartilhadas dos diferentes } \\
\text { saberes interagem nas disciplinas, convergindo para o processo interativo rumo } \\
\text { à visão integral do indivíduo. }\end{array}$ \\
\hline $\begin{array}{l}\text { Martins, Barreto \& Pordeus. } \\
\text { Características associadas ao uso de } \\
\text { serviços odontológicos entre idosos } \\
\text { dentados e desdentados no Sudeste } \\
\text { do Brasil: Projeto SB Brasil, } 2008 .\end{array}$ & $\begin{array}{l}\text { Investigou-se o uso de serviços odontológicos entre idosos dentados e } \\
\text { desdentados residentes na Região Sudeste do Brasil. A análise mostrou diferenças } \\
\text { entre dentados e desdentados. O menor uso entre idosos que mais necessitam } \\
\text { sugere iniquidade no acesso aos serviços odontológicos. }\end{array}$ \\
\hline $\begin{array}{l}\text { Rosa et al. Odontogeriatria: a saúde } \\
\text { bucal na terceira idade, } 2008 \text {. }\end{array}$ & $\begin{array}{l}\text { S autores realizam revisão de literatura sobre saúde bucal e Geriatria e fazem } \\
\text { onsiderações sobre o atendimento clínico a essa faixa etária, ressaltando as } \\
\text { rincipais carências envolvidas na saúde bucal para essa população. }\end{array}$ \\
\hline $\begin{array}{l}\text { Mesas, Trelha \& Azevedo. Saúde } \\
\text { bucal de idosos restritos ao domicílio: } \\
\text { estudo descritivo de uma demanda } \\
\text { interdisciplinar, } 2008 \text {. }\end{array}$ & $\begin{array}{l}\text { Pesquisa realizada em município brasileiro, objetivando verificar a condição de } \\
\text { idosos restritos ao domicílio. Devido às precárias condições encontradas, serão } \\
\text { necessários estudos considerando a restrição ao domićlio e suas implicações, } \\
\text { como fatores de risco para a má condição e manutenção da saúde bucal em idosos. }\end{array}$ \\
\hline $\begin{array}{l}\text { Benedetti, Melo \& Gonçalves. Idosos } \\
\text { de Florianópolis: autopercepção das } \\
\text { condições de saúde bucal e utilização } \\
\text { de serviços odontológicos, } 2007 \text {. }\end{array}$ & $\begin{array}{l}\text { Os dados apresentados na pesquisa são relativos à autopercepção dos entrevistados } \\
\text { sobre suas condições de saúde bucal e uso dos serviços odontológicos, que foram } \\
\text { associados com as variáveis sociodemográficas. O estudo permite concluir pela } \\
\text { exigência de políticas públicas de atenção específica à saúde bucal dos idosos, } \\
\text { de modo a promover saúde e bem-estar para todos, no irreversível processo de } \\
\text { envelhecer. }\end{array}$ \\
\hline $\begin{array}{l}\text { Benedetti et al. Tendência na } \\
\text { utilização de serviços odontológicos } \\
\text { entre idosos brasileiros e fatores } \\
\text { associados: um estudo baseado na } \\
\text { Pesquisa Nacional por Amostra de } \\
\text { Domicílios (1998 e 2003), } 2007 \text {. }\end{array}$ & $\begin{array}{l}\text { O objetivo deste estudo foi determinar a prevalência e os fatores associados } \\
\text { ao uso de serviços odontológicos entre idosos brasileiros em } 1998 \text { e } 2003 \text {. } \\
\text { Observam-se importantes desigualdades sociais associadas ao uso de serviços } \\
\text { odontológicos por idosos, apontando para a necessidade premente de políticas } \\
\text { que visem à redução dessas desigualdades. }\end{array}$ \\
\hline
\end{tabular}




\begin{tabular}{|c|c|}
\hline Autores, títulos e ano de publicação & Objetivos e principais resultados \\
\hline $\begin{array}{l}\text { Martins, Barreto \& Pordeus. Uso de } \\
\text { serviços odontológicos entre idosos } \\
\text { brasileiros, } 2007 \text {. }\end{array}$ & $\begin{array}{l}\text { O objetivo foi investigar a utilização de serviços odontológicos e seus } \\
\text { determinantes entre idosos brasileiros. A saúde bucal dos idosos brasileiros } \\
\text { é precária e o uso de serviços odontológicos foi menor entre os que mais } \\
\text { necessitavam. Diferentes fatores estiveram associados ao uso entre dentados e } \\
\text { desdentados. Apenas a escolaridade, o acesso a informações sobre saúde bucal } \\
\text { e a dor nos dentes ou gengiva nos últimos três meses foram comuns aos dois } \\
\text { estratos. São necessários investimentos públicos em saúde bucal para reverter } \\
\text { esse quadro. }\end{array}$ \\
\hline $\begin{array}{l}\text { Campostrini, Ferreira \& Rocha. } \\
\text { Condições da saúde bucal do idoso } \\
\text { brasileiro, } 2007 .\end{array}$ & $\begin{array}{l}\text { Do ponto de vista da saúde pública, para mudar o quadro são esperadas ações } \\
\text { preventivas, assistenciais e de reabilitação de acordo com o propósito da Política } \\
\text { Nacional de Saúde do Idoso vinculada às demais políticas públicas em vigor. }\end{array}$ \\
\hline $\begin{array}{l}\text { Santos et al. Autopercepção em saúde } \\
\text { bucal de idosos em unidades de saúde } \\
\text { da família do Distrito Sanitário III de } \\
\text { João Pessoa-PB, } 2007 .\end{array}$ & $\begin{array}{l}\text { O objetivo deste estudo foi avaliar a autopercepção em saúde bucal em idosos } \\
\text { não institucionalizados. Sugere-se a implementação de medidas de promoção } \\
\text { à saúde direcionadas à população idosa, no intuito de disseminar informações } \\
\text { sobre saúde bucal e fortalecendo o vínculo da equipe de saúde família enquanto } \\
\text { agente promotor de saúde. }\end{array}$ \\
\hline $\begin{array}{l}\text { Hebling, Rodrigues. O estatuto do } \\
\text { idoso e a saúde bucal, } 2006 \text {. }\end{array}$ & $\begin{array}{l}\text { O objetivo do trabalho foi descrever os direitos estabelecidos por essa lei } \\
\text { relacionados à saúde bucal dos idosos. Ações públicas de atenção e a promoção } \\
\text { de saúde bucal aos idosos devem ser adequadas à nova legislação. }\end{array}$ \\
\hline $\begin{array}{l}\text { Monti, et al. Análise comparada da } \\
\text { saúde bucal do idoso na cidade de } \\
\text { Araçatuba, } 2006 .\end{array}$ & $\begin{array}{l}\text { Com base nas políticas públicas de saúde de atenção ao idoso, preconizadas pelo } \\
\text { Ministério da Saúde, o artigo analisa a atenção à saúde bucal do idoso, ilustrando } \\
\text { uma realidade brasileira quanto à falta de tratamentos preventivos, curativos e } \\
\text { educacionais. }\end{array}$ \\
\hline $\begin{array}{l}\text { Unfer et al. Autopercepção da perda } \\
\text { de dentes em idosos, } 2006 \text {. }\end{array}$ & $\begin{array}{l}\text { O objetivo do trabalho foi analisar as percepções de um grupo de idosos sobre } \\
\text { a perda de dentes. Torna-se imprescindível o desenvolvimento de iniciativas no } \\
\text { campo da educação e prevenção em saúde bucal, enfatizando ações voltadas para } \\
\text { a atenção integral do idoso, destacando-se a dimensão social das doenças e o papel } \\
\text { do Estado como provedor da saúde e da qualidade de vida de todos os cidadãos. }\end{array}$ \\
\hline $\begin{array}{l}\text { Silva et al. Saúde bucal e autopercepção } \\
\text { em adultos e idosos de Piracicaba-SP, } \\
2006 .\end{array}$ & $\begin{array}{l}\text { Diante dos resultados obtidos, há a necessidade de programas preventivos e } \\
\text { educativos tanto para idosos como para adultos, para que estes cheguem à terceira } \\
\text { idade com uma condição de saúde bucal melhor que a relatada atualmente. }\end{array}$ \\
\hline $\begin{array}{l}\text { Moreira et al. A Saúde bucal do idoso } \\
\text { brasileiro: revisão sistemática sobre o } \\
\text { quadro epidemiológico e acesso aos } \\
\text { serviços de saúde bucal, } 2005 \text {. }\end{array}$ & $\begin{array}{l}\text { A saúde bucal do idoso brasileiro encontra-se em situação precária, com } \\
\text { elevados índices de edentulismo, refletindo a ineficácia historicamente presente } \\
\text { nos serviços públicos de atenção odontológica, limitado a extrações em série e } \\
\text { serviços de urgência, baseados no modelo curativista. }\end{array}$ \\
\hline $\begin{array}{l}\text { Saintrain, Souza. Saúde bucal do } \\
\text { idoso: desafio a ser perseguido, } 2005 .\end{array}$ & $\begin{array}{l}\text { Este texto é pertinente em destacar a construção da política de saúde do idoso } \\
\text { no Brasil e a preocupação da sua efetividade em garantir e assegurar de modo } \\
\text { universal e com equidade os serviços e benefícios a que fazem jus. }\end{array}$ \\
\hline $\begin{array}{l}\text { Matos, Giatti \& Lima-Costa. Fatores } \\
\text { sociodemográficos associados ao } \\
\text { uso de serviços odontológicos entre } \\
\text { idosos brasileiros: um estudo baseado } \\
\text { na Pesquisa Nacional por Amostra de } \\
\text { Domicílios, } 2004 \text {. }\end{array}$ & $\begin{array}{l}\text { Os resultados do trabalho mostram que os idosos brasileiros apresentam } \\
\text { baixa taxa de uso de serviços odontológicos, e que diferenças regionais e } \\
\text { socioeconômicas são importantes para determinar a frequência de uso de } \\
\text { serviços odontológicos entre idosos brasileiros. }\end{array}$ \\
\hline
\end{tabular}




\begin{tabular}{|c|c|}
\hline Autores, títulos e ano de publicação & Objetivos e principais resultados \\
\hline $\begin{array}{l}\text { Colussi, } \quad \text { Freitas. Aspectos } \\
\text { epidemiológicos da saúde do idoso no } \\
\text { Brasil, 2002. }\end{array}$ & $\begin{array}{l}\text { A falta de padronização na organização e apresentação dos dados, assim como } \\
\text { a sua escassez, prejudicaram a análise do trabalho, sugerindo que deva haver } \\
\text { maior preocupação, por parte dos pesquisadores, quanto à realização de novas } \\
\text { pesquisas, e principalmente, quanto à maneira como estas serão conduzidas. } \\
\text { Apesar disso, confirmaram-se as condições muito precárias de saúde bucal em } \\
\text { idosos no Brasil. }\end{array}$ \\
\hline $\begin{array}{l}\text { Meneghim, Pereira \& Silva. } \\
\text { Prevalência de cárie radicular e } \\
\text { condição periodontal em uma } \\
\text { população idosa institucionalizada de } \\
\text { Piracicaba-SP, } 2002 \text {. }\end{array}$ & $\begin{array}{l}\text { Constatou-se que há aumento na prevalência de lesões de cárie e outras afecções } \\
\text { radiculares, como erosão e abrasão, indicando aumento na susceptibilidade a tais } \\
\text { lesões com o passar da idade, justificando-se assim a necessidade de políticas } \\
\text { voltadas para essa parcela da população. }\end{array}$ \\
\hline $\begin{array}{l}\text { Silva \& Valsecki Júnior. Avaliação das } \\
\text { condições de saúde bucal dos idosos } \\
\text { em um município brasileiro, } 2000 .\end{array}$ & $\begin{array}{l}\text { Os resultados mostram o comprometimento da qualidade de vida de grande } \\
\text { parte dessas pessoas e indicam a necessidade de maior atenção dos serviços } \\
\text { públicos a esse grupo populacional. }\end{array}$ \\
\hline
\end{tabular}

\section{DISCUSSÃO}

Epidemiologia da saúde bucal em idosos: algumas notas

Os artigos selecionados, focando diferentes realidades do nosso país, apontam para a precária condição de saúde bucal desse grupo populacional. Assim, analisando os dados encontrados, constata-se a atual necessidade de tratamento odontológico pelos idosos, seja para reposição de próteses ou para tratamento de alguma afecção bucal. ${ }^{9}$

Esse fato reafirma que no Brasil é baixo o relato de uso dos serviços odontológicos por idosos, uma vez que $6,3 \%$ relataram nunca terem utilizado os serviços odontológicos, que somente 13,2\% utilizaram os serviços há menos de um ano e que $80,5 \%$ utilizaram o serviço há um ano ou mais. ${ }^{10}$ Afirma-se que $56,5 \%$ dos idosos não iam ao dentista há mais de três anos. Quanto a idosos restritos ao domicílio, $79 \%$ relataram não ter acesso aos serviços odontológicos há mais de cinco anos. ${ }^{11}$

$\mathrm{O}$ acesso à atenção em saúde bucal é necessário para manter a saúde bucal, prevenir o edentulismo, e também para melhorar a qualidade de vida dos idosos, sejam eles dentados ou desdentados. No caso dos idosos desdentados, o uso de serviços odontológicos é importante para avaliar a necessidade de utilização ou substituição de próteses dentárias e para o diagnóstico precoce de lesões potencialmente cancerígenas. ${ }^{10,12}$ Resultados das pesquisas epidemiológicas sobre a saúde bucal do idoso no Brasil demonstram dados preocupantes quando se referem à universalidade e à equidade de acesso e uso dos serviços de saúde bucal. ${ }^{13}$

Estudos comprovam que há pouca procura pelos serviços de saúde bucal por idosos, o que pode estar relacionado com a falta de percepção da real situação de saúde oral desse grupo. A autoavaliação é positiva, mas contraditória: mesmo tendo uma saúde bucal ruim, a maioria dos idosos faz uma boa avaliação sobre sua saúde bucal.,114-16 $\mathrm{O}$ adoecer, as péssimas condições de saúde bucal e o edentulismo são aceitos como condição natural e normal do indivíduo que envelhece. ${ }^{3}$ Assim, observa-se que o uso dos serviços odontológicos por idosos se reduz conforme aumenta a idade. ${ }^{10}$ Essa cultura acaba refletindo em menores cuidados com a saúde bucal. ${ }^{15}$

As diferenças entre a autoavaliação e as condições objetivas de saúde bucal são provavelmente a principal razão para os indivíduos não procurarem os serviços odontológicos quando estes estão disponíveis. ${ }^{15}$ 
Além do fator cultural, outros fatores levam à iniquidade no acesso e uso dos serviços odontológicos, como aspectos sociais e econômicos, baixo nível de escolaridade, baixa renda, ${ }^{3,10,12}$ residir em área rural ${ }^{12} \mathrm{e}$ ainda a pouca oferta de serviços públicos. ${ }^{3}$ Indivíduos dentados usam mais os serviços que os desdentados, sendo que o principal motivo são os problemas bucais relacionados à dor, ${ }^{9,12}$ podendo indicar o uso dos serviços para exodontias de dentes remanescentes e comprometidos por cárie e/ou doença periodontal, acentuando o edentulismo. ${ }^{12}$ Os idosos desdentados acreditam não necessitar de serviços odontológicos, pois já não possuem dentes. Dessa forma, informações quanto à prevenção de patologias bucais, como cáries, perda dental e câncer bucal não chegam até essa população. ${ }^{11}$

Atualmente, os idosos carregam a herança de um modelo assistencial centrado na doença com práticas curativas e mutiladoras, ${ }^{3} \mathrm{o}$ que resultou em um quadro atual precário, com idosos apresentando grande quantidade de problemas bucais, como dentes extraídos, sextantes com doença periodontal, lesões da mucosa bucal e necessidade de próteses e, consequentemente, grande demanda por serviços protéticos para essa população. $3,10,13,17,18$

Outros autores sugerem que a condição dentária dos idosos não seja explicada pela exposição a tratamentos inadequados, visto que a oferta de serviços odontológicos era pouco frequente quando esses idosos eram crianças e jovens, fase essencial para uma dentição sadia, e que alguns idosos jamais usaram esses serviços. ${ }^{10,12}$ Vale lembrar, também, que os idosos atuais não foram expostos à fluoretação da água e da pasta dental, o que com certeza concorreu para a precariedade de sua saúde bucal. ${ }^{3}$

Analisando os artigos com dados epidemiológicos, ${ }^{3,4,10-12,17-20}$ os resultados referentes ao índice de dentes cariados, perdidos e obturados (CPOD) médio encontrado foi de $28,02 \%$, sendo que o componente extraído foi o mais prevalente, variando de $66 \%$ a 93\%. A prevalência do edentulismo ficou em $67,39 \%$. O uso da prótese total é mais frequente no arco superior do que no inferior. Quando o estudo envolve idosos institucionalizados ou restritos ao domicílio, a situação ainda é mais precária com CPOD, chegando a $30,91 \%{ }^{15}$ e $29,7 \%,{ }^{16}$ respectivamente.

A histórica escassez de atenção odontológica a essa faixa etária resultou na necessidade de formulação de políticas públicas para promoção de saúde bucal para pessoas idosas, para que elas experimentem essa época da vida com qualidade. $3,10,13,14,19$

\section{A abordagem à saúde bucal do idoso: propostas para o cotidiano do SUS}

Conforme demonstrado, as condições de saúde bucal do idoso não são boas e tendem a se agravar caso não sejam tomadas medidas de atenção a essa população. Para tal, entendese que o atendimento ao paciente idoso requer ações interdisciplinares. ${ }^{16,21-23}$ Fisicamente, o envelhecimento leva a alterações fisiológicas e/ou patológicas. Psicologicamente, o idoso apresenta uma carga emotiva acumulada ao longo dos anos. $^{21-22}$ Todos os profissionais da equipe de saúde da família devem observar as condições de saúde bucal dos idosos, independentemente de sua formação, e as situações que levantem dúvidas devem ser esclarecidas com o cirurgião-dentista responsável pela área de abrangência. ${ }^{16}$ Por outro ângulo, é necessário que o cirurgião-dentista considere o impacto das condições sistêmicas sobre a saúde bucal, sendo necessária a avaliação de profissionais de outras áreas para prestar um atendimento odontológico integral ao idoso. ${ }^{23,24}$

A precariedade da saúde bucal do idoso pode ser notada tanto pelo quadro epidemiológico como pela ausência de programas específicos para esse grupo populacional. Nesse contexto, poderiam ser exploradas as ações de educação enfatizando a autopercepção e, através disso, conscientizar o grupo da necessidade de cuidados com a sua saúde bucal. ${ }^{1} \mathrm{O}$ edentulismo não deve ser aceito como um fenômeno normal e natural que acompanha o envelhecimento. Políticas preventivas de saúde devem ser criadas e destinadas à população adulta para a manutenção dos dentes até idades avançadas. ${ }^{4}$ 
O modelo assistencial de atenção à saúde bucal do idoso deve seguir os princípios do SUS com destaque para a integralidade, equidade e universalidade, estando ainda de acordo com as condições técnico-administrativas do município. Para tanto, é necessário conhecer e compreender a realidade desses idosos por meio de estudos epidemiológicos e ações intersetoriais, estabelecendo a partir daí as prioridades necessárias para traçar as estratégias de mudanças no modelo de assistência, onde o idoso possa assumir seu papel de agente transformador de sua própria saúde. ${ }^{3}$

Com a inserção da saúde bucal na ESF e a implantação do Programa Brasil Sorridente pelo Ministério da Saúde, surge uma nova perspectiva de melhorar a situação de saúde bucal da população idosa. As Equipes de Saúde Bucal na Estratégia Saúde da Família (ESB/ESF) podem ofertar serviços de atenção à saúde bucal do idoso como tratamento das alterações de tecidos moles, com ênfase na detecção de lesões cancerizáveis, ${ }^{25}$ o tratamento ou remoção de dentes em precárias condições, o tratamento periodontal, a reabilitação protética e considerações sobre promoção e prevenção da saúde bucal. No entanto, faz-se necessário que os profissionais das ESB/ESF identifiquem os idosos nas suas áreas de abrangência, direcionando ações específicas para esse grupo populacional. ${ }^{6}$ Acrescenta-se a isso o fato de que a Odontologia não pode adotar uma postura de omissão frente a essa realidade, uma vez que suas ações específicas são importantes para que se conforme o princípio da integralidade, contribuindo para a qualidade de vida de imensos contingentes populacionais.

O paciente idoso é um indivíduo que vivenciou várias experiências e passou por diversas mudanças ao longo de sua vida e, apesar da sabedoria adquirida, várias limitações lhe são impostas, tanto funcionais quanto psicossociais. ${ }^{24}$ Portanto, o atendimento odontológico ao paciente deve ser observado sob diversos aspectos. Há uma relação direta entre o tratamento odontológico e as manifestações sistêmicas. Com isso, o cuidado com os idosos deve ser diferenciado. Essa complexidade deve ser atendida por uma equipe interdisciplinar de profissionais, visando melhorar a qualidade de vida dos pacientes idosos e proporcionar um envelhecimento saudável. Viver mais e bem está intimamente relacionado à saúde tanto geral como odontológica. ${ }^{21,22,24-26}$

Mesmo com as políticas públicas atuais, os idosos não são capazes de perceber a relação entre a saúde geral e a saúde bucal. Os profissionais da ESF devem compreender o idoso como um todo indissociável, atendendo-o na sua totalidade no que se refere ao estado de saúde (bucal e geral). A ESF visa à integralidade - portanto, a formação de seus membros deve ser voltada para a interdisciplinaridade. ${ }^{27}$

Considerando os princípios do SUS, a atenção à saúde bucal do idoso deve ser "assegurada pelo SUS desde a prevenção, terapia das doenças bucais, seja em ambiente ambulatorial, consultórios ou clínicas de referência, ou em ambiente domiciliar com o uso de equipamentos odontológico transportável". ${ }^{27}$ Nas equipes de ESF, as visitas domiciliares da ESB às pessoas acamadas ou com dificuldades de locomoção devem ser organizadas, ampliando e qualificando as ações de saúde bucal, objetivando identificar os riscos e proporcionando o acompanhamento e tratamentos necessários. ${ }^{16,27}$

$\mathrm{Na}$ literatura pesquisada, ${ }^{5,10,12,15,23}$ há um consenso quanto a organizar a atenção à saúde bucal do idoso, devendo a equipe de saúde bucal oferecer ações educativas, preventivas, de recuperação e reabilitação da saúde bucal, visando a mudanças no quadro epidemiológico desses indivíduos como também qualidade de vida.

\section{CONCLUSÃO}

A leitura dos artigos selecionados aponta para a necessidade de um planejamento inter e multidisciplinar capaz de responder aos desafios da atenção à saúde bucal de idosos. $\mathrm{O}$ processo fisiológico do envelhecimento, aliado às possíveis alterações sistêmicas a que o grupo está sujeito, justifica essa escolha. O necessário 
apoio intersetorial é apontado e pode contribuir sobremaneira para a promoção da saúde.

As equipes devem buscar reconhecer nos idosos em suas áreas de abrangência aqueles que apresentem demandas de saúde bucal. Acredita-se que a atenção à saúde bucal prestada pelas atuais Equipes de Saúde Bucal na Estratégia Saúde da Família em muitos municípios necessita de uma reformulação, direcionando suas ações para os problemas específicos da população idosa em suas áreas de abrangência. Essas ações vão desde o planejamento, levantamentos epidemiológicos, ações de prevenção e promoção da saúde bucal, como também ações de restauração e reabilitação.
Diante do exposto, propõe-se que sejam incluídas no processo de trabalho das Equipes de Saúde Bucal na Estratégia Saúde da Família ações de atenção à saúde bucal do idoso.

Tal escolha não é aleatória, uma vez que se entende que são essas equipes que devem possuir competência necessária para a implementação de ações que possibilitem a ruptura com o modelo assistencial hegemônico (hospitalocêntrico, curativista, reducionista, biologicista) e permitam que práticas baseadas na promoção da saúde, ampliadas, usuário-centradas, possam ser uma realidade no dia a dia do sistema público de saúde que se busca construir.

\section{REFERÊNCIAS}

1. Instituto Brasileiro de Geografia e Estatística. Indicadores Sociodemográficos e de saúde no Brasil, 2009. Rio de Janeiro: IBGE; 2009. A Dinâmica demográfica brasileira e os impactos nas políticas públicas; 18 folhas. (Estudos e pesquisas. Informação Demográfica e Socioeconômica, 25).

2. Gordilho A, Sérgio J, Silvestre J, Ramos LR, Freire MPA, Espindola N, et al. Desafios a serem enfrentados no terceiro milênio pelo setor saúde na atenção integral do idoso. Rio de Janeiro: UERJ, UnATI; 2000.

3. Moreira RS, Nico LS, Tomita NE, Ruiz T. A saúde bucal do idoso brasileiro: revisão sistemática sobre o quadro epidemiológico e acesso aos serviços de saúde bucal. Cad Saúde Pública 2005;21(6):1665-75.

4. Colussi C, Freitas SFT. Aspectos epidemiológicos da saúde bucal do idoso no Brasil. Cad Saúde Pública 2002;18(5):1313-20.

5. Brasil. Ministério da Saúde. Diretrizes operacionais dos pactos pela vida, em defesa do SUS e de gestão. Brasília, DF: Ministério da Saúde; 2006. (Série A. Normas e manuais técnicos).

6. Brasil. Portaria GM/MS no. 1.444, de 28 de dezembro de 2000. Portaria de incentivos financeiros. Diário Oficial da União de 29 dez. 2000, seção 1, p.85.

7. Brasil. Ministério da Saúde, Secretaria de Atenção à Saúde, Departamento de Atenção Básica, Coordenação Nacional de Saúde Bucal. Diretrizes da política nacional de saúde bucal. Brasília, DF: Ministério da Saúde; 2004.
8. Bernardo WM, Nobre MRC, Jatene FB. A prática clínica baseada em evidências. Parte II: buscando as evidências em fontes de informação. Rev Assoc Med Bras 2004;50(1):1-9.

9. Costa IMD, Maciel SML, Cavalcanti AL. Acesso aos serviços odontológicos e motivos da procura por atendimento por pacientes idosos em Campina Grande - PB. Odontol Clín-Cient 2010;7(4): 331-5.

10. Matos DL, Giatti L, Lima-Costa MF. Fatores sócio-demográficos associados ao uso de serviços odontológicos entre idosos brasileiros: um estudo baseado na Pesquisa Nacional por Amostra de Domicílios. Cad Saúde Pública 2004;20(5):1290-7.

11. Silva AL, Saintrain MVL. Interferência do perfil epidemiológico do idoso na atenção odontológica. Rev Bras Epidemiol 2006;9(2):242-50.

12. Martins AMEBL, Haikal DS, Pereira SM, Barreto SM. Uso de serviços odontológicos por rotina entre idosos brasileiros: Projeto SB Brasil. Cad Saúde Pública 2008;24(7):1651-66.

13. Saintrain MVL, Souza EHA. Saúde bucal do idoso: Desafio a ser perseguido. Odontol Clín-Cient 2005;4(2):152-60.

14. Benedetti TRB, Mello ALSF, Gonçalves LHT. Idosos de Florianópolis: autopercepção das condições de saúde bucal e utilização de serviços odontológicos. Ciênc Saúde Coletiva 2007;12(6):1683-90.

15. Martins AMEBL, Barreto SM, Pordeus IA. Autoavaliação de saúde bucal em idosos: análise com base em modelo multidimensional. Cad Saúde Pública 2009;25(2):421-35. 
16. Mesas AE, Trelha CS, Azevedo MJ. Saúde bucal de idosos restritos ao domicílio: estudo descritivo de uma demanda interdisciplinar. Physis 2008;18(1):61-75.

17. Colussi C, Freitas SFT. Aspectos epidemiológicos da saúde bucal do idoso no Brasil. Cad Saúde Pública 2002;18(5):1313-20

18. Silva SRC, Valsecki A Júnior. Avaliação das condições de saúde bucal dos idosos em um município brasileiro. Rev Panam Salud Publica 2000;8(4):198-210.

19. Matos DL, Lima-Costa MF. Tendência na utilização de serviços odontológicos entre idosos brasileiros e fatores associados: um estudo baseado na Pesquisa Nacional por Amostra de Domicílios (1998 e 2003). Cad Saúde Pública 2007;23(11):2740-8.

20. Silva AL, Saintrain MVL. Interferência do perfil epidemiológico do idoso na atenção odontológica. Rev Bras Epidemiol 2006;9(2):242-50.

21. Macedo DN, Carvalho SS, Lira SS, Sena CA, Bezerra EAD. Proposta de um protocolo para o atendimento odontológico do paciente idoso na atenção básica. Odontol Clín-Cient 2005;8(3):257-62.
22. Rosa LB, Zuccolotto MCC, Bataglion C, Coronatto EAS. Odontogeriatria: a saúde bucal na terceira idade. RFO UPF 2008;13(2):82-6.

23. Saintrain ML, Vieira LJES. Saúde bucal do idoso: abordagem interdisciplinar. Ciênc Saúde Coletiva 2008;13(4):1127-32.

24. Silva EMM, Da Silva CE Filho, Fajardo RS, Fernandes AUR, Marchiori AV. Mudanças fisiológicas e psicológicas na velhice relevantes no tratamento odontológico. Rev Ciênc Ext 2005;2(1):72-5.

25. Shinkai RSA, Cury AADB. O papel da odontologia na equipe interdisciplinar: contribuindo para a atenção integral ao idoso. Cad Saúde Pública 2000;16(4):1099-1109.

26. Santos FB. Autopercepção em saúde bucal de idosos em unidades de saúde da família do Distrito Sanitário III de João Pessoa-PB. Arq Odontol 2007;43(2):435-50.

27. Hebling E, Rodrigues KC. O Estatuto do Idoso e a saúde bucal. ROBRAC 2006;15(39):51-6. 